\title{
Limits on Light Gravitino Production and New Processes with Large Missing Transverse Energy in $p \bar{p}$ Collisions at $\sqrt{s}=1.8 \mathrm{TeV}$
}

T. Affolder, ${ }^{21}$ H. Akimoto, ${ }^{43}$ A. Akopian, ${ }^{36}$ M. G Albrow, ${ }^{10}$ P. Amaral, ${ }^{7}$ S. R. Amendolia, ${ }^{32}$ D. Amidei, ${ }^{24}$ K. Anikeev ${ }^{22}$ J. Antos, ${ }^{1}$ G. Apollinari, ${ }^{36}$ T. Arisawa,${ }^{43}$ T. Asakawa,${ }^{41}$ W. Ashmanskas, ${ }^{7}$ M. Atac,${ }^{10}$ F. Azfar, ${ }^{29}$ P. Azzi-Bacchetta ${ }^{30}$ N. Bacchetta, ${ }^{30}$ M. W. Bailey, ${ }^{26}$ S. Bailey, ${ }^{14}$ P. de Barbaro, ${ }^{35}$ A. Barbaro-Galtieri, ${ }^{21}$ V. E. Barnes,${ }^{34}$ B. A. Barnett, ${ }^{17}$ M. Barone, ${ }^{12}$ G. Bauer, ${ }^{22}$ F. Bedeschi, ${ }^{32}$ S. Belforte, ${ }^{40}$ G. Bellettini, ${ }^{32}$ J. Bellinger, ${ }^{44}$ D. Benjamin, ${ }^{9}$ J. Bensinger, ${ }^{4}$

A. Beretvas, ${ }^{10}$ J.P. Berge, ${ }^{10}$ J. Berryhill, ${ }^{7}$ B. Bevensee, ${ }^{31}$ A. Bhatti, ${ }^{36}$ M. Binkley, ${ }^{10}$ D. Bisello, ${ }^{30}$ R. E. Blair ${ }^{2}$ C. Blocker, ${ }^{4}$ K. Bloom, ${ }^{24}$ B. Blumenfeld, ${ }^{17}$ S. R. Blusk, ${ }^{35}$ A. Bocci, ${ }^{32}$ A. Bodek,,${ }^{35}$ W. Bokhari, ${ }^{31}$ G. Bolla, ${ }^{34}$ Y. Bonushkin, ${ }^{5}$ D. Bortoletto, ${ }^{34}$ J. Boudreau, ${ }^{33}$ A. Brandl, ${ }^{26}$ S. van den Brink, ${ }^{17}$ C. Bromberg, ${ }^{25}$ M. Brozovic, ${ }^{9}$

N. Bruner ${ }^{26}$ E. Buckley-Geer, ${ }^{10}$ J. Budagov, ${ }^{8}$ H. S. Budd, ${ }^{35}$ K. Burkett, ${ }^{14}$ G. Busetto, ${ }^{30}$ A. Byon-Wagner, ${ }^{10}$ K. L. Byrum, ${ }^{2}$ M. Campbell, ${ }^{24}$ W. Carithers, ${ }^{21}$ J. Carlson, ${ }^{24}$ D. Carlsmith,${ }^{44}$ J. Cassada, ${ }^{35}$ A. Castro, ${ }^{30}$ D. Cauz, ${ }^{40}$ A. Cerri, ${ }^{32}$ A. W. Chan, ${ }^{1}$ P. S. Chang, ${ }^{1}$ P. T. Chang, ${ }^{1}$ J. Chapman, ${ }^{24}$ C. Chen,${ }^{31}$ Y. C. Chen, ${ }^{1}$ M.-T. Cheng, ${ }^{1}$ M. Chertok, ${ }^{38}$ G. Chiarelli, ${ }^{32}$ I. Chirikov-Zorin, ${ }^{8}$ G. Chlachidze, ${ }^{8}$ F. Chlebana, ${ }^{10}$ L. Christofek, ${ }^{16}$ M. L. Chu, ${ }^{1}$ S. Cihangir, ${ }^{10}$ C. I. Ciobanu, ${ }^{27}$ A. G. Clark, ${ }^{13}$ A. Connolly, ${ }^{21}$ J. Conway,${ }^{37}$ J. Cooper, ${ }^{10}$ M. Cordelli, ${ }^{12}$ J. Cranshaw, ${ }^{39}$ D. Cronin-Hennessy, ${ }^{9}$ R. Cropp, ${ }^{23}$ R. Culbertson, ${ }^{7}$ D. Dagenhart, ${ }^{42}$ F. DeJongh,,${ }^{10}$ S. Dell' Agnello, ${ }^{12}$ M. Dell'Orso, ${ }^{32}$ R. Demina, ${ }^{10}$ L. Demortier, ${ }^{36}$ M. Deninno, ${ }^{3}$ P. F. Derwent, ${ }^{10}$ T. Devlin, ${ }^{37}$ J. R. Dittmann, ${ }^{10}$ S. Donati, ${ }^{32}$ J. Done,${ }^{38}$ T. Dorigo, ${ }^{14}$ N. Eddy,${ }^{16}$ K. Einsweiler, ${ }^{21}$ J. E. Elias, ${ }^{10}$ E. Engels, Jr. ${ }^{33}$ W. Erdmann, ${ }^{10}$ D. Errede, ${ }^{16}$ S. Errede, ${ }^{16}$ Q. Fan, ${ }^{35}$ R. G. Feild, ${ }^{45}$ C. Ferretti, ${ }^{32}$ R. D. Field,${ }^{11}$ I. Fiori, ${ }^{3}$ B. Flaugher, ${ }^{10}$ G. W. Foster, ${ }^{10}$ M. Franklin, ${ }^{14}$ J. Freeman,,${ }^{10}$ J. Friedman, ${ }^{22}$ Y. Fukui ${ }^{20}$ S. Galeotti, ${ }^{32}$ M. Gallinaro, ${ }^{36}$ T. Gao, ${ }^{31}$ M. Garcia-Sciveres, ${ }^{21}$ A. F. Garfinkel,,${ }^{34}$ P. Gatti, ${ }^{30}$ C. Gay, ${ }^{45}$ S. Geer, ${ }^{10}$ D. W. Gerdes,${ }^{24}$ P. Giannetti, ${ }^{32}$ P. Giromini, ${ }^{12}$ V. Glagolev,${ }^{8}$ M. Gold,${ }^{26}$ J. Goldstein,,${ }^{10}$ A. Gordon, ${ }^{14}$ A. T. Goshaw, ${ }^{9}$ Y. Gotra, ${ }^{33}$ K. Goulianos,${ }^{36}$ C. Green, ${ }^{34}$ L. Groer, ${ }^{37}$ C. Grosso-Pilcher, ${ }^{7}$ M. Guenther, ${ }^{34}$ G. Guillian, ${ }^{24}$ J. Guimaraes da Costa, ${ }^{24}$ R. S. Guo, ${ }^{1}$ C. Haber ${ }^{21}$ E. Hafen, ${ }^{22}$ S. R. Hahn, ${ }^{10}$ C. Hall, ${ }^{14}$ T. Handa, ${ }^{15}$ R. Handler, ${ }^{44}$ W. Hao,${ }^{39}$ F. Happacher, ${ }^{12}$ K. Hara, ${ }^{41}$ A. D. Hardman, ${ }^{34}$ R. M. Harris, ${ }^{10}$ F. Hartmann, ${ }^{18}$ K. Hatakeyama, ${ }^{36}$ J. Hauser, ${ }^{5}$ J. Heinrich, ${ }^{31}$ A. Heiss, ${ }^{18}$ M. Herndon, ${ }^{17}$ B. Hinrichsen, ${ }^{23}$ K. D. Hoffman, ${ }^{34}$ C. Holck, ${ }^{31}$ R. Hollebeek, ${ }^{31}$ L. Holloway, ${ }^{16}$

R. Hughes,${ }^{27}$ J. Huston, ${ }^{25}$ J. Huth, ${ }^{14}$ H. Ikeda,${ }^{41}$ J. Incandela, ${ }^{10}$ G. Introzzi,,${ }^{32}$ J. Iwai, ${ }^{43}$ Y. Iwata, ${ }^{15}$ E. James, ${ }^{24}$ H. Jensen, ${ }^{10}$ M. Jones, ${ }^{31}$ U. Joshi, ${ }^{10}$ H. Kambara, ${ }^{13}$ T. Kamon, ${ }^{38}$ T. Kaneko, ${ }^{41}$ K. Karr, ${ }^{42}$ H. Kasha, ${ }^{45}$ Y. Kato ${ }^{28}$ T. A. Keaffaber, ${ }^{34}$ K. Kelley, ${ }^{22}$ M. Kelly,${ }^{24}$ R. D. Kennedy, ${ }^{10}$ R. Kephart, ${ }^{10}$ D. Khazins, ${ }^{9}$ T. Kikuchi, ${ }^{41}$ M. Kirk, ${ }^{4}$ B. J. Kim, ${ }^{19}$ H. S. Kim,${ }^{16}$ M. J. Kim ${ }^{19}{ }^{\text {S. H. Kim }},{ }^{41}$ Y. K. Kim, ${ }^{21}$ L. Kirsch, ${ }^{4}$ S. Klimenko, ${ }^{11}$ P. Koehn, ${ }^{27}$ A. Köngeter, ${ }^{18}$ K. Kondo, ${ }^{43}$ J. Konigsberg, ${ }^{11}$ K. Kordas, ${ }^{23}$ A. Korn, ${ }^{22}$ A. Korytov, ${ }^{11}$ E. Kovacs, ${ }^{2}$ J. Kroll,,${ }^{31}$ M. Kruse,${ }^{35}$

S. E. Kuhlmann, ${ }^{2}$ K. Kurino, ${ }^{15}$ T. Kuwabara, ${ }^{41}$ A. T. Laasanen, ${ }^{34}$ N. Lai, ${ }^{7}$ S. Lami, ${ }^{36}$ S. Lammel,${ }^{10}$ J. I. Lamoureux, ${ }^{4}$ M. Lancaster, ${ }^{21}$ G. Latino, ${ }^{32}$ T. LeCompte, ${ }^{2}$ A. M. Lee IV,${ }^{9}$ S. Leone, ${ }^{32}$ J. D. Lewis, ${ }^{10}$ M. Lindgren, ${ }^{5}$ T. M. Liss, ${ }^{16}$ J. B. Liu, ${ }^{35}$ Y. C. Liu, ${ }^{1}$ N. Lockyer, ${ }^{31}$ J. Loken, ${ }^{29}$ M. Loreti, ${ }^{30}$ D. Lucchesi,${ }^{30}$ P. Lukens, ${ }^{10}$ S. Lusin,${ }^{44}$ L. Lyons, ${ }^{29}$ J. Lys, ${ }^{21}$ R. Madrak, ${ }^{14}$ K. Maeshima,${ }^{10}$ P. Maksimovic, ${ }^{14}$ L. Malferrari,${ }^{3}$ M. Mangano, ${ }^{32}$ M. Mariotti, ${ }^{30}$ G. Martignon, ${ }^{30}$ A. Martin, ${ }^{45}$ J. A. J. Matthews, ${ }^{26}$ J. Mayer, ${ }^{23}$ P. Mazzanti, ${ }^{3}$ K. S. McFarland, ${ }^{35}$ P. McIntyre, ${ }^{38}$ E. McKigney, ${ }^{31}$ M. Menguzzato, ${ }^{30}$ A. Menzione, ${ }^{32}$ C. Mesropian, ${ }^{36}$ T. Miao, ${ }^{10}$ R. Miller ${ }^{25}$ J. S. Miller, ${ }^{24}$ H. Minato,${ }^{41}$ S. Miscetti, ${ }^{12}$ M. Mishina, ${ }^{20}$ G. Mitselmakher, ${ }^{11}$ N. Moggi, ${ }^{3}$ E. Moore,${ }^{26}$ R. Moore,${ }^{24}$ Y. Morita, ${ }^{20}$ A. Mukherjee, ${ }^{10}$ T. Muller, ${ }^{18}$ A. Munar, ${ }^{32}$ P. Murat, ${ }^{10}$ S. Murgia,${ }^{25}$ M. Musy ${ }^{40}$ J. Nachtman, ${ }^{5}$ S. Nahn,${ }^{45}$ H. Nakada, ${ }^{41}$ T. Nakaya, ${ }^{7}$ I. Nakano, ${ }^{15}$

C. Nelson, ${ }^{10}$ D. Neuberger ${ }^{18}$ C. Newman-Holmes, ${ }^{10}$ C.-Y.P. Ngan, ${ }^{22}$ P. Nicolaidi, ${ }^{40}$ H. Niu, ${ }^{4}$ L. Nodulman, ${ }^{2}$ A. Nomerotski, ${ }^{11}$ S.H. Oh, ${ }^{9}$ T. Ohmoto, ${ }^{15}$ T. Ohsugi, ${ }^{15}$ R. Oishi, ${ }^{41}$ T. Okusawa, ${ }^{28}$ J. Olsen, ${ }^{44}$ C. Pagliarone, ${ }^{32}$ F. Palmonari, ${ }^{32}$ R. Paoletti, ${ }^{32}$ V. Papadimitriou, ${ }^{39}$ S. P. Pappas,${ }^{45}$ D. Partos,${ }^{4}$ J. Patrick, ${ }^{10}$ G. Pauletta,${ }^{40}$ M. Paulini, ${ }^{21}$ C. Paus,${ }^{22}$ L. Pescara,${ }^{30}$ T. J. Phillips, ${ }^{9}$ G. Piacentino, ${ }^{32}$ K. T. Pitts,${ }^{16}$ R. Plunkett,${ }^{10}$ A. Pompos,${ }^{34}$ L. Pondrom,${ }^{44}$ G. Pope,${ }^{33}$ M. Popovic, ${ }^{23}$ F. Prokoshin, ${ }^{8}$ J. Proudfoot, ${ }^{2}$ F. Ptohos, ${ }^{12}$ G. Punzi, ${ }^{32}$ K. Ragan, ${ }^{23}$ A. Rakitine,${ }^{22}$ D. Reher, ${ }^{21}$ A. Reichold,${ }^{29}$ W. Riegler, ${ }^{14}$ A. Ribon, ${ }^{30}$ F. Rimondi, ${ }^{3}$ L. Ristori, ${ }^{32}$ W. J. Robertson, ${ }^{9}$ A. Robinson, ${ }^{23}$ T. Rodrigo, ${ }^{6}$ S. Rolli, ${ }^{42}$ L. Rosenson, ${ }^{22}$ R. Roser, ${ }^{10}$ R. Rossin, ${ }^{30}$ W. K. Sakumoto, ${ }^{35}$ D. Saltzberg, ${ }^{5}$ A. Sansoni, ${ }^{21}$ L. Santi, ${ }^{40}$ H. Sato, ${ }^{41}$ P. Savard, ${ }^{23}$ P. Schlabach, ${ }^{10}$ E. E. Schmidt, ${ }^{10}$ M. P. Schmidt, ${ }^{45}$ M. Schmitt, ${ }^{14}$ L. Scodellaro, ${ }^{30}$ A. Scott, ${ }^{5}$

A. Scribano, ${ }^{32}$ S. Segler, ${ }^{10}$ S. Seidel, ${ }^{26}$ Y. Seiya, ${ }^{41}$ A. Semenov, ${ }^{8}$ F. Semeria, ${ }^{3}$ T. Shah,${ }^{22}$ M. D. Shapiro,${ }^{21}$

P. F. Shepard, ${ }^{33}$ T. Shibayama, ${ }^{41}$ M. Shimojima, ${ }^{41}$ M. Shochet, ${ }^{7}$ J. Siegrist,${ }^{21}$ G. Signorelli, ${ }^{32}$ A. Sill, ${ }^{39}$ P. Sinervo, ${ }^{23}$

P. Singh, ${ }^{16}$ A. J. Slaughter, ${ }^{45}$ K. Sliwa ${ }^{42}$ C. Smith, ${ }^{17}$ F. D. Snider, ${ }^{10}$ A. Solodsky, ${ }^{36}$ J. Spalding, ${ }^{10}$ T. Speer, ${ }^{13}$ P. Sphicas, ${ }^{22}$ F. Spinella, ${ }^{32}$ M. Spiropulu,,${ }^{14}$ L. Spiegel, ${ }^{10}$ J. Steele, ${ }^{44}$ A. Stefanini, ${ }^{32}$ J. Strologas, ${ }^{16}$ F. Strumia, ${ }^{13}$ 
D. Stuart, ${ }^{10}$ K. Sumorok, ${ }^{22}$ T. Suzuki, ${ }^{41}$ T. Takano, ${ }^{28}$ R. Takashima,${ }^{15}$ K. Takikawa, ${ }^{41}$ P. Tamburello, ${ }^{9}$ M. Tanaka, ${ }^{41}$ B. Tannenbaum, ${ }^{5}$ W. Taylor ${ }^{23}$ M. Tecchio,${ }^{24}$ P. K. Teng, ${ }^{1} \mathrm{~K}$. Terashi, ${ }^{41} \mathrm{~S}$. Tether, ${ }^{22}$ D. Theriot, ${ }^{10} \mathrm{R}$. Thurman-Keup, ${ }^{2}$ P. Tipton, ${ }^{35} \mathrm{~S}$. Tkaczyk, ${ }^{10} \mathrm{~K}$. Tollefson, ${ }^{35}$ A. Tollestrup, ${ }^{10} \mathrm{H}$. Toyoda, ${ }^{28} \mathrm{~W}$. Trischuk, ${ }^{23} \mathrm{~J} . \mathrm{F}$. de Troconiz, ${ }^{14} \mathrm{~J}$. Tseng,${ }^{22}$ N. Turini, ${ }^{32}$ F. Ukegawa, ${ }^{41}$ T. Vaiciulis,${ }^{35}$ J. Valls,${ }^{37}$ S. Vejcik III,,${ }^{10}$ G. Velev,${ }^{10}$ R. Vidal,${ }^{10}$ R. Vilar, ${ }^{6}$ I. Volobouev, ${ }^{21}$ D. Vucinic, ${ }^{22}$ R. G. Wagner, ${ }^{2}$ R. L. Wagner, ${ }^{10}$ J. Wahl, ${ }^{7}$ N. B. Wallace, ${ }^{37}$ A. M. Walsh, ${ }^{37}$ C. Wang, ${ }^{9}$ C. H. Wang, ${ }^{1}$ M. J. Wang, ${ }^{1}$ T. Watanabe, ${ }^{41}$ D. Waters, ${ }^{29}$ T. Watts, ${ }^{37}$ R. Webb, ${ }^{38}$ H. Wenzel,,${ }^{18}$ W. C. Wester III, ${ }^{10}$ A. B. Wicklund,${ }^{2}$ E. Wicklund, ${ }^{10}$ H. H. Williams, ${ }^{31}$ P. Wilson, ${ }^{10}$ B. L. Winer, ${ }^{27}$ D. Winn, ${ }^{24}$ S. Wolbers, ${ }^{10}$ D. Wolinski, ${ }^{24}$ J. Wolinski, ${ }^{25}$ S. Wolinski, ${ }^{24}$ S. Worm,${ }^{26}$ X. Wu, ${ }^{13}$ J. Wyss, ${ }^{32}$ A. Yagil, ${ }^{10}$ W. Yao, ${ }^{21}$ G. P. Yeh, ${ }^{10}$ P. Yeh, ${ }^{1}$ J. Yoh, ${ }^{10}$ C. Yosef, ${ }^{25}$ T. Yoshida, ${ }^{28}$ I. Yu, ${ }^{19}$ S. Yu, ${ }^{31}$ A. Zanetti, ${ }^{40}$ F. Zetti, ${ }^{21}$ and S. Zucchelli ${ }^{3}$

(CDF Collaboration)

${ }^{1}$ Institute of Physics, Academia Sinica, Taipei, Taiwan 11529, Republic of China

${ }^{2}$ Argonne National Laboratory, Argonne, Illinois 60439

${ }^{3}$ Istituto Nazionale di Fisica Nucleare, University of Bologna, I-40127 Bologna, Italy

${ }^{4}$ Brandeis University, Waltham, Massachusetts 02254

${ }^{5}$ University of California at Los Angeles, Los Angeles, California 90024

${ }^{6}$ Instituto de Fisica de Cantabria, University of Cantabria, 39005 Santander, Spain

${ }^{7}$ Enrico Fermi Institute, University of Chicago, Chicago, Illinois 60637

${ }^{8}$ Joint Institute for Nuclear Research, RU-141980 Dubna, Russia

${ }^{9}$ Duke University, Durham, North Carolina 27708

${ }^{10}$ Fermi National Accelerator Laboratory, Batavia, Illinois 60510

${ }^{11}$ University of Florida, Gainesville, Florida 32611

${ }^{12}$ Laboratori Nazionali di Frascati, Istituto Nazionale di Fisica Nucleare, I-00044 Frascati, Italy

${ }^{13}$ University of Geneva, CH-1211 Geneva 4, Switzerland

${ }^{14}$ Harvard University, Cambridge, Massachusetts 02138

${ }^{15}$ Hiroshima University, Higashi-Hiroshima 724, Japan

${ }^{16}$ University of Illinois, Urbana, Illinois 61801

${ }^{17}$ The Johns Hopkins University, Baltimore, Maryland 21218

${ }^{18}$ Institut für Experimentelle Kernphysik, Universität Karlsruhe, 76128 Karlsruhe, Germany

${ }^{19}$ Korean Hadron Collider Laboratory, Kyungpook National University, Taegu 702-701, Korea, Seoul National University, Seoul 151-742, Korea,

and SungKyunKwan University, Suwon 440-746, Korea

${ }^{20}$ High Energy Accelerator Research Organization (KEK), Tsukuba, Ibaraki 305, Japan

${ }^{21}$ Ernest Orlando Lawrence Berkeley National Laboratory, Berkeley, California 94720

${ }^{22}$ Massachusetts Institute of Technology, Cambridge, Massachusetts 02139

${ }^{23}$ Institute of Particle Physics, McGill University, Montreal, Canada H3A 2T8 and University of Toronto, Toronto, Canada M5S $1 A 7$

${ }^{24}$ University of Michigan, Ann Arbor, Michigan 48109

${ }^{25}$ Michigan State University, East Lansing, Michigan 48824

${ }^{26}$ University of New Mexico, Albuquerque, New Mexico 87131

${ }^{27}$ The Ohio State University, Columbus, Ohio 43210

${ }^{28}$ Osaka City University, Osaka 588, Japan

${ }^{29}$ University of Oxford, Oxford OX1 3RH, United Kingdom

${ }^{30}$ Universita di Padova, Istituto Nazionale di Fisica Nucleare, Sezione di Padova, I-35131 Padova, Italy

${ }^{31}$ University of Pennsylvania, Philadelphia, Pennsylvania 19104

${ }^{32}$ Istituto Nazionale di Fisica Nucleare, University and Scuola Normale Superiore of Pisa, I-56100 Pisa, Italy

${ }^{33}$ University of Pittsburgh, Pittsburgh, Pennsylvania 15260

${ }^{34}$ Purdue University, West Lafayette, Indiana 47907

${ }^{35}$ University of Rochester, Rochester, New York 14627

${ }^{36}$ Rockefeller University, New York, New York 10021

${ }^{37}$ Rutgers University, Piscataway, New Jersey 08855

${ }^{38}$ Texas A\&M University, College Station, Texas 77843

${ }^{39}$ Texas Tech University, Lubbock, Texas 79409

${ }^{40}$ Istituto Nazionale di Fisica Nucleare, University of Trieste, Udine, Italy

${ }^{41}$ University of Tsukuba, Tsukuba, Ibaraki 305, Japan

${ }^{42}$ Tufts University, Medford, Massachusetts 02155

${ }^{43}$ Waseda University, Tokyo 169, Japan

${ }^{44}$ University of Wisconsin, Madison, Wisconsin 53706

${ }^{45}$ Yale University, New Haven, Connecticut 06520 
(Received 21 March 2000)

Events collected by the Collider Detector at Fermilab (CDF) with an energetic jet plus large missing transverse energy can be used to search for physics beyond the standard model. We see no deviations from the expected backgrounds and set upper limits on the production of new processes. We consider in addition the production of light gravitinos and set a limit at $95 \%$ confidence level on the breaking scale $\sqrt{F} \geq 217 \mathrm{GeV}$, which excludes gravitino masses smaller than $1.1 \times 10^{-5} \mathrm{eV} / c^{2}$.

PACS numbers: 13.85.Rm, 12.60.Jv, 13.87.Ce, 14.80.Ly

In $p \bar{p}$ collisions, undetectable particles manifest themselves as missing transverse energy, $\not_{T}$. Events characterized by large amounts of $\mathscr{E}_{T}$ are interesting for searches of physics beyond the standard model. Supersymmetry, for instance, relates each bosonic/fermionic standard model particle to a fermionic/bosonic superpartner, providing a solution to the hierarchy problem [1]. In models with spontaneous breaking of the global supersymmetry, the goldstino, a massless and neutral spin- $\frac{1}{2}$ particle, is introduced. When gravitation is added and supersymmetry is realized locally the gauge particle, graviton, has a spin- $\frac{3}{2}$ partner, the gravitino $(\tilde{G})$, which acquires a mass, $m_{\tilde{G}}$, while the goldstino is absorbed [2].

At the Tevatron, gravitinos can be produced in pairs in association either with a jet according to the processes $q \bar{q} \rightarrow \tilde{G} \tilde{G} g, q g \rightarrow \tilde{G} \tilde{G} q, \bar{q} g \rightarrow \tilde{G} \tilde{G} \bar{q}$, and $g g \rightarrow \tilde{G} \tilde{G} g$, or with a photon following $q \bar{q} \rightarrow \tilde{G} \tilde{G} \gamma$. In the scenario in which all other supersymmetric particles are heavy, the only parameter upon which these processes depend is the supersymmetry-breaking scale $\sqrt{F}$ [3] and the cross sections vary as $1 / m_{\tilde{G}}^{4}$ (or as $1 / F^{4}$ ) [4]. If supersymmetry is present, and the gravitino is very light $\left(m_{\tilde{G}} \ll\right.$ $10^{-4} \mathrm{eV} / c^{2}$ ), it can be seen at the Tevatron by looking at final states which include gravitinos and ordinary particles only [4]. In this case the lightest supersymmetric particle is the gravitino which escapes undetected manifesting itself as $\not_{T}$.

We present, in this paper, cross section limits for processes with an energetic jet plus large $\mathbb{E}_{T}$. This signature is characteristic of processes not described by the standard model, such as the production of light gravitino pairs plus one jet [4]. The data sample used for this analysis was collected with the CDF detector from 1994 to 1995, and corresponds to a total integrated luminosity of $87 \mathrm{pb}^{-1}$. The CDF detector is described in detail elsewhere [5]; only features essential to this analysis are summarized here. The momenta of charged particles are measured in the central tracking chamber (CTC), which is inside a $1.4 \mathrm{~T}$ superconducting solenoidal magnet. Outside the CTC, electromagnetic and hadronic calorimeters, which are segmented in $\eta-\phi$ towers and cover the pseudorapidity region $|\eta|<4.2$ [6], are used to identify jets and electron candidates. Outside the calorimeters, drift chambers in the region $|\eta|<1.0$ provide muon identification.

Events for this analysis passed a multilevel trigger system which selected events with $\mathbb{E}_{T} \geq 35 \mathrm{GeV}$. $\mathbb{E}_{T}$ is defined to be the magnitude of the vector sum of transverse energy in all calorimeter towers with $|\eta| \leq 3.6$
[7]. Such a trigger has an efficiency of about $80 \%$ for events with $\mathbb{E}_{T} \geq 50 \mathrm{GeV}$ and becomes fully efficient above $80 \mathrm{GeV}$. After removing cosmic ray and accelerator related backgrounds [8] we select events with $\mathscr{E}_{T} \geq 50 \mathrm{GeV}$, at least one jet [9] with transverse energy $E_{T} \geq 10 \mathrm{GeV}$ in the central region, $|\eta| \leq 0.7$, and with the additional requirement of $E_{T} \geq 80 \mathrm{GeV}$ and $|\eta| \leq 2.4$ for the most energetic jet. These requirements define the topology we are looking for and reduce the presence of unphysical backgrounds.

The backgrounds expected from standard model sources are due to $W+$ jet or $Z+$ jet processes plus a small contribution from $t \bar{t}$ and diboson $(W W, W Z, Z Z)$ production, and are dominated by events containing neutrinos from $W$ or $Z$ decays. We estimate these with the PYTHIA [10] generator and a full simulation of the CDF detector. The cross sections for the $W / Z+$ jet processes are taken, for each value of jet multiplicity, from CDF measurements [11,12]. The cross sections for $t \bar{t}$ and diboson processes are taken from theory $[13,14]$. The contribution from all these processes is reduced by rejecting events containing electrons or muons with large transverse momentum, $P_{T}: P_{T} \geq 10 \mathrm{GeV} / c$ for electrons, $P_{T} \geq 5 \mathrm{GeV} / c$ for muons, or $P_{T} \geq 10 \mathrm{GeV} / c$ if the muon is within $\Delta R \equiv \sqrt{(\Delta \eta)^{2}+(\Delta \phi)^{2}}=1$ from a jet. Additional rejection is obtained by removing events which contain a jet with a ratio of electromagnetic to total energy larger than 0.95 or isolated tracks of $P_{T} \geq 30 \mathrm{GeV} / c$, the latter requirement aimed at reducing tau sources. Here an isolated track is defined as a track for which the $\sum P_{T}$ of additional tracks within a cone of radius $\Delta R=0.4$ is smaller than $10 \mathrm{GeV} / c$. A total of 16019 events pass these requirements.

The resulting data sample is dominated by instrumental backgrounds, due to mismeasurement of otherwise balanced QCD multijet events. The behavior of these backgrounds is studied using a control sample passing a trigger which selected $1 / 40$ of the events having at least one jet with $E_{T} \geq 50 \mathrm{GeV}$. Apart from prescaling, the kinematical requirements we impose guarantee full overlap with the signal sample. To reduce the instrumental backgrounds we cut on the azimuthal angle, $\Delta \phi\left(\mathbb{E}_{T}, j\right)$, between the direction of $\mathbb{E}_{T}$ and the nearest jet. The distributions of $\Delta \phi\left(\mathbb{E}_{T}, j\right)$ for the data, the control sample, and for the standard model processes considered are shown in Fig. 1. The requirement $\Delta \phi\left(\mathscr{E}_{T}, j\right) \geq 1.57 \mathrm{rad}$ is very effective in removing the instrumental backgrounds: from an extrapolation of the 


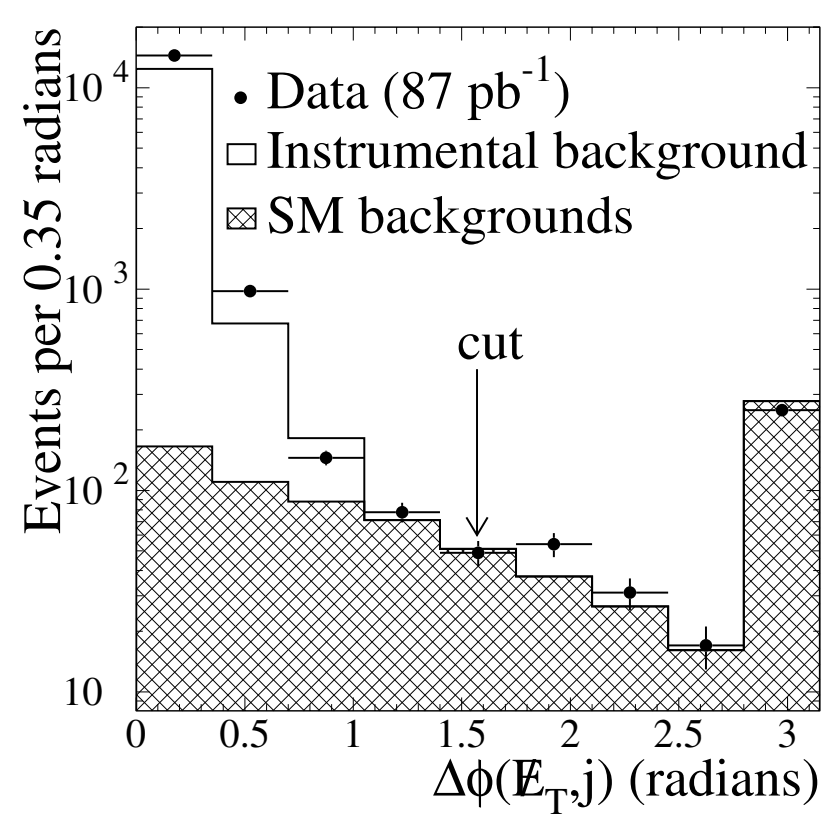

FIG. 1. The distribution of $\Delta \phi\left(\mathscr{E}_{T}, j\right)$ for events before the $\Delta \phi$ cut is applied (points), compared to the instrumental backgrounds inferred from the control sample (white area) and the standard model backgrounds (shaded area).

behavior of the control sample, and loosening the $\mathbb{E}_{T}$ cut to populate the tails of the distribution, we derive that at most 14 events (at 95\% confidence level, C.L.) from instrumental backgrounds are expected to survive this cut with respect to a total of 379 events selected. The number of events expected from standard model processes is $380 \pm 129$, with contributions mainly from $Z+$ jet $(204 \pm 69)$ and $W+$ jet $(171 \pm 57)$ processes. Backgrounds from cosmic rays or beam halo have been considered and found negligible [8] ( $\leq 4$ events at 95\% C.L.). One source of uncertainty on the background estimate derives from uncertainties on the production cross sections (20\% for $W / Z+$ jet cross sections [11,12], 20\% for $t \bar{t}$ corresponding to the range of theoretical calculations [13], and $30 \%$ for diboson production related to the use of different sets of parton distribution functions [14]). Another contribution comes from the uncertainty on the selection acceptance $(25 \%)$ due mainly to the uncertainty on the jet energy scale (from $5 \%$ for low- $E_{T}$ jets to $3 \%$ for high- $E_{T}$ ones, reflecting the uncertainty in our knowledge of the reconstructed jet energy [9]). A minor contribution of $4 \%$ comes from the uncertainty on the integrated luminosity.

A jet correction algorithm [15] is applied to $\mathbb{E}_{T}$ which takes into account calorimeter nonlinearities and reduced response at boundaries between modules and calorimeter subsystems. No correction is applied for high- $P_{T}$ muons since we remove the events containing them. Figure 2 shows the $\mathbb{E}_{T}$ distribution; the data and the expectation for standard model processes are in good agreement. The 95\% C.L. upper limits on the product of acceptance times cross section for the production of physics beyond the stan-

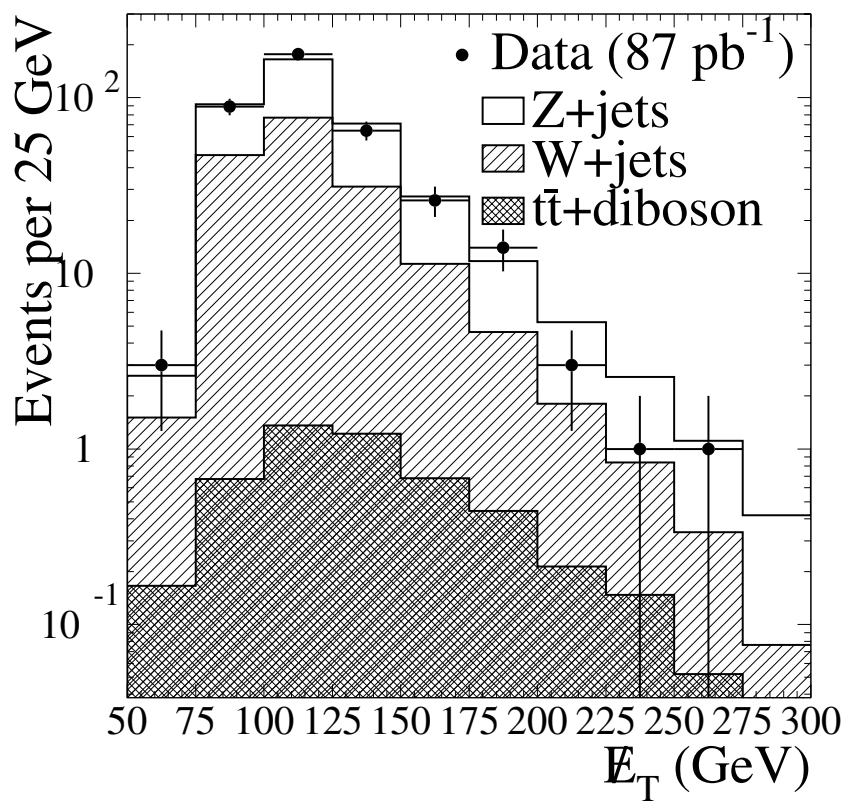

FIG. 2. The distribution of $\mathbb{E}_{T}$ for data (points) compared to the central value expectations from $Z+$ jet events (white area), $W+$ jet events (shaded area), and for $t \bar{t}+W W+W Z+Z Z$ events (hatched area).

dard model are obtained using a Monte Carlo technique [16] which convolutes the uncertainties on the integrated luminosity with background expectations. The limits, as a function of the $\mathbb{E}_{T}$ threshold, $\mathbb{E}_{T}^{\min }$, are shown in Fig. 3. Systematic uncertainties on the acceptance are not included because they depend on the particular physics process under consideration.

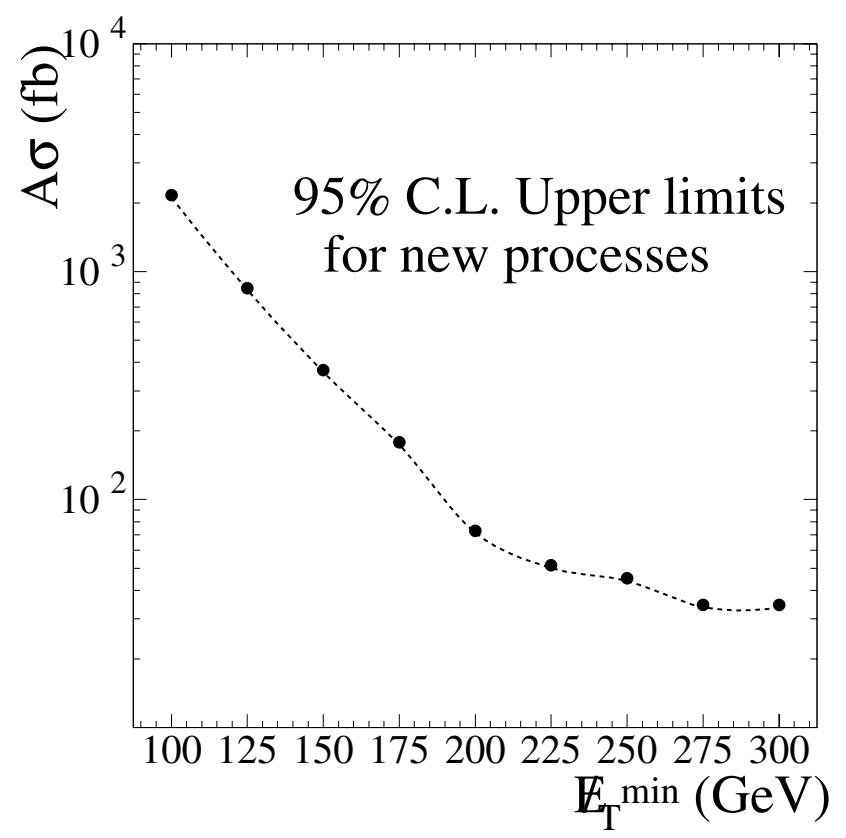

FIG. 3. The $95 \%$ C.L. upper limits on the product of acceptance $(A)$ times cross section $(\sigma)$ for the production of new processes. 
The production of gravitinos, $p \bar{p} \rightarrow \tilde{G} \tilde{G} g, \tilde{G} \tilde{G} q$, is simulated in HERWIG [17] by including the calculated matrix elements [4], followed by a detector simulation. For the generation the following inputs are used: $\sqrt{F}=200 \mathrm{GeV}$; factorization/renormalization scale, $\mu$, equal to the transverse energy of the emitted quark/ gluon; and the MRSD' set of parton distribution functions [18]. For such a choice of parameters, the production cross section, evaluated for $P_{T}^{\tilde{G}} \tilde{G} \geq 100 \mathrm{GeV} / c$, amounts to $12.6 \pm 4.0 \mathrm{pb}$, where $P_{T}^{\tilde{G}} \tilde{G}$ is the transverse magnitude of the vector sum of the two gravitino momenta before any further radiation has occurred. The uncertainty on the cross section has several contributions which are added in quadrature: (i) $30 \%$ due to the choice of factorization/ renormalization scale ( $\mu=2 E_{T}$ vs $\mu=E_{T} / 2$ ); (ii) $10 \%$ due to the gluon radiation modeling in the Monte Carlo, obtained by comparing the cross sections before and after radiation occurs; (iii) and 5\% due to the choice of parton distribution function (e.g., MRSD' vs CTEQ2M [19]). The signal acceptance is the fraction of events with $P_{T}^{\tilde{G} \tilde{G}} \geq 100 \mathrm{GeV} / c$ which pass the selection with a cut on $\not_{T}$.

We use the Monte Carlo technique mentioned above and convolute the uncertainty on the acceptance with background estimates to derive the upper limit on the production cross section for $\tilde{G} \tilde{G}+$ jet events with $P_{T}^{\tilde{G} \tilde{G}} \geq 100 \mathrm{GeV} / c$ (see Fig. 4). The best sensitivity (i.e., the smallest expected upper limit on the cross section) is reached for $\mathbb{E}_{T}^{\min }=175 \mathrm{GeV}$. For such a threshold, the acceptance amounts to $(6.2 \pm 1.2) \%$, where the uncer-

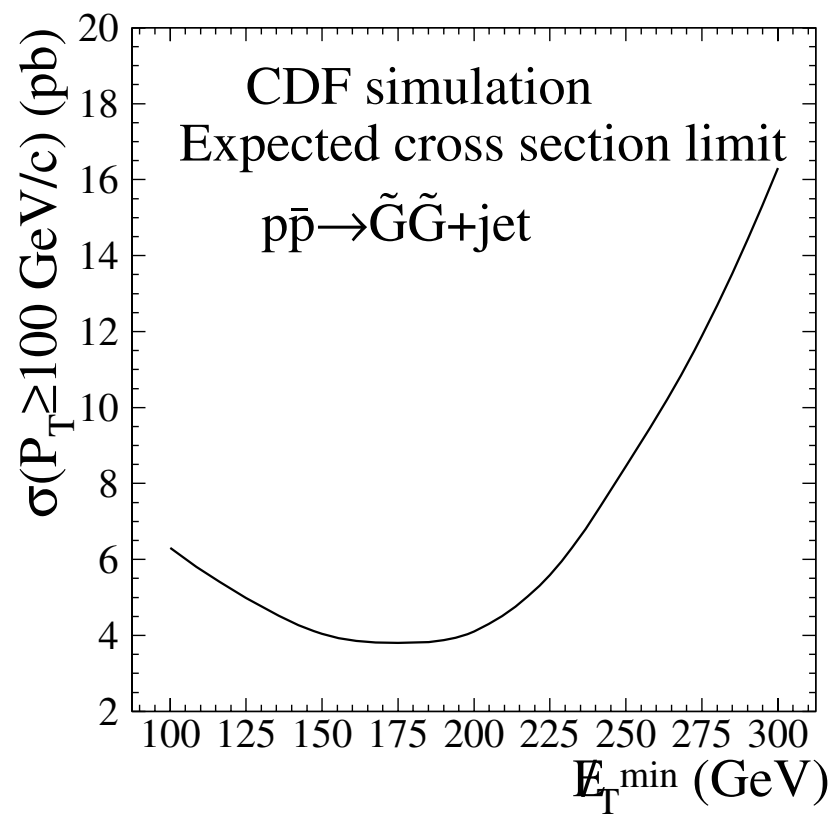

FIG. 4. The 95\% C.L. upper limit on the cross section for $\tilde{G} \tilde{G}+$ jet events with $P_{T}^{\tilde{G} \tilde{G}} \geq 100 \mathrm{GeV} / c$ expected for no signal. tainty is dominated by the choice of absolute energy scale and the modeling of initial or final state gluon radiation. Apart from the $\mathscr{E}_{T}$ threshold, the other selection criteria have a relative efficiency of about $80 \%$, essentially due to the requirement of a central jet and to the $\Delta \phi\left(\mathbb{E}_{T}, j\right)$ cut. With 19 events selected above the optimized $\mathscr{E}_{T}$ threshold of $175 \mathrm{GeV}$ and an estimated background of $21.6 \pm 7.0$ events, we derive the $95 \%$ C.L. upper limit on the signal of 16.9 events, accounting for the $20 \%$ relative uncertainty on the acceptance. This signal corresponds to an upper limit on the production cross section of $3.1 \mathrm{pb}$ for $P_{T}^{\tilde{G}} \tilde{G} \geq 100 \mathrm{GeV} / c$. In an ensemble of pseudoexperiments with the expected background and no true signal we would obtain this limit or better $35 \%$ of the time. Considering the $1 / F^{4}$ dependence of the production cross section we derive, from the comparison to the theory, a 95\% C.L. limit $\sqrt{F} \geq 217 \mathrm{GeV}$. Such a limit corresponds to a gravitino mass larger than $1.1 \times 10^{-5} \mathrm{eV} / c^{2}$. We note that these limits are independent of any unmeasured parameters; they would become stronger if other supersymmetric particles were produced.

In conclusion, we have compared events containing large $\mathscr{E}_{T}$ and at least one energetic jet to the expectations from standard model processes and instrumental backgrounds. The $\mathbb{E}_{T}$ distribution has been used to derive upper limits on the product of acceptance times cross section for the production of new processes beyond the standard model. We have selected 19 events with $\mathbb{E}_{T} \geq 175 \mathrm{GeV}$ with respect to an estimated background of $21.6 \pm 7.0$ events. This implies a 95\% C.L. upper limit on the cross section of $3.1 \mathrm{pb}$ for the production of $\tilde{G} \tilde{G}+$ jet events with $P_{T}^{\tilde{G} \tilde{G}} \geq 100 \mathrm{GeV} / c$. Comparing this number to the theoretical cross section we have derived the 95\% C.L. limit $\sqrt{F} \geq 217 \mathrm{GeV}$, which corresponds to a gravitino mass $m_{\tilde{G}} \geq 1.1 \times 10^{-5} \mathrm{eV} / c^{2}$. This limit is comparable to LEP measurements $\left[(0.8-1.0) \times 10^{-5} \mathrm{eV} / c^{2}\right]$ [20] based on events with photons and missing energy.

We thank the Fermilab staff and the technical staff of the participating institutions for their vital contributions. This work was supported by the U.S. Department of Energy and National Science Foundation; the Italian Istituto Nazionale di Fisica Nucleare; the Ministry of Education, Science, Sports and Culture of Japan; the Natural Sciences and Engineering Research Council of Canada; the National Science Council of the Republic of China; the Swiss National Science Foundation; the A. P. Sloan Foundation; the Bundesministerium fuer Bildung und Forschung, Germany; and the Korea Science and Engineering Foundation.

[1] For reviews, see H. P. Nilles, Phys. Rep. 110, 1 (1984); H. E. Haber and G. L. Kane, Phys. Rep. 117, 75 (1985); S. P. Martin, hep-ph/9709356; G. L. Kane, Perspective on Supersymmetry (World Scientific, Singapore, 1998). 
[2] E. Cremmer, B. Julia, J. Scherk, S. Ferrara, L. Girardello, and P. van Nieuwenhuizen, Nucl. Phys. B147, 105 (1979).

[3] $F=\sqrt{3} m_{\tilde{G}} \bar{M}_{P}$, where $\quad \bar{M}_{P}=\left(8 \pi G_{N}\right)^{-1 / 2}=2.4 \times$ $10^{18} \mathrm{GeV} / c^{2}$ is the reduced Planck mass.

[4] A. Brignole, F. Feruglio, M. L. Mangano, and F. Zwirner, Nucl. Phys. B526, 136 (1998). A revised version can be found in hep-ph/9801329.

[5] F. Abe et al., Nucl. Instrum. Methods Phys. Res., Sect. A 271, 387 (1988); F. Abe et al., Phys. Rev. D 50, 2966 (1994).

[6] In the CDF coordinate system, the $z$ axis lies along the beam line. The pseudorapidity $\eta$ is defined as $-\ln \tan (\theta / 2)$, where $\theta$ is the polar angle with respect to the proton beam direction. The azimuthal angle with respect to the $z$ axis is denoted by $\phi$.

[7] F. Abe et al., Phys. Rev. D 43, 2070 (1991).

[8] M. Spiropulu, Ph.D. thesis, Harvard University (unpublished).

[9] Jets are defined as clusters of energy in the calorimeter found with a fixed-cone algorithm with $\Delta R \equiv$ $\sqrt{(\Delta \eta)^{2}+(\Delta \phi)^{2}}=0.7$. See, e.g., F. Abe et al., Phys. Rev. D 45, 1448 (1992).

[10] T. Sjöstrand, Comput. Phys. Commun. 82, 74 (1994). We use version 5.7 .
[11] F. Abe et al., Phys. Rev. Lett. 79, 4760 (1997).

[12] F. Abe et al., Phys. Rev. Lett. 77, 448 (1996).

[13] E. Laenen, J. Smith, and W. L. van Neerven, Phys. Lett. B 321, 254 (1994); S. Catani, M. L. Mangano, P. Nason, and L. Trentadue, Phys. Lett. B 378, 329 (1996); E. L. Berger and H. Contopanagos, Phys. Rev. D 54, 3085 (1996).

[14] J. Ohnemus and J. F. Owens, Phys. Rev. D 43, 3626 (1991); J. Ohnemus, Phys. Rev. D 44, 1403 (1991); 44, 3477 (1991); B. Mele, P. Nason, and G. Ridolfi, Nucl. Phys. B357, 409 (1991); S. Frixione, P. Nason, and G. Ridolfi, Nucl. Phys. B383, 3 (1992); S. Frixione, Nucl. Phys. B410, 280 (1993).

[15] F. Abe et al., Phys. Rev. D 47, 4857 (1993).

[16] F. Abe et al., Phys. Rev. D 56, R1357 (1997).

[17] G. Marchesini et al., Comput. Phys. Commun. 67, 465 (1992). We use version 5.6.

[18] A. D. Martin, R. G. Roberts, and W. J. Stirling, Phys. Lett. B 306, 145 (1993).

[19] H. L. Lai et al., Phys. Rev. D 51, 4763 (1995).

[20] R. Barate et al., Phys. Lett. B 429, 201 (1998); P. Abreu et al., Report No. CERN-EP-2000-021 (to be published in Eur. Phys. J. C); M. Acciarri et al., Phys. Lett. B 470, 268 (1999). 